\title{
Riesgo de infección de mascotas con COVID-19 desde la perspectiva de sus propietarios en Lima, Perú
}

\author{
Risk of infection of COVID-19 in pets from the perspective of their \\ owners in Lima, Peru
}

\author{
Gonzalo Villegas ${ }^{1}$, Daphne León ${ }^{1}$, Néstor Falcón ${ }^{1,2}$
}

\section{Resumen}

El objetivo del estudio fue evaluar la percepción de los propietarios de mascotas acerca del riesgo de infección de sus animales con COVID-19 en Lima, Perú. Se elaboró una encuesta en formato virtual dirigida a clientes de servicios veterinarios particulares de Lima Metropolitana. El instrumento recabó información demográfica, conocimientos sobre transmisión del SARS-CoV-2, casos de infección humana con dicho virus, manejo de la mascota en pandemia y percepción sobre el riesgo de infección de los animales por COVID-19. La encuesta fue respondida por 408 propietarios. Se reconoció que el principal medio de transmisión del COVID-19 eran las personas infectadas (93.6\%). Los problemas respiratorios fueron poco observados en perros $(8.1 \%)$ y gatos $(2.2 \%)$. La percepción mayoritaria de los encuestados fue el no relacionar los problemas respiratorios de las mascotas con COVID-19 (80.6\%), pero mostraron preocupación por la salud afectada de su animal (79.7\%). La práctica de paseo de las mascotas se realizaba más de una vez al día (38.1\%), siendo más frecuente no utilizar protección para los animales (65.4\%). El paseo duraba generalmente entre 10 y 20 minutos (45.4\%), realizándolo alrededor de la manzana (46.2\%) y en parques $(36.0 \%)$. Las medidas de higiene y prevención tomadas tras el paseo fueron baños y lavados de patas (73.4\%) en las mascotas, y combinación de medidas que incluyeron distanciamiento social (80.4\%), uso de equipos de protección

\footnotetext{
${ }^{1}$ Laboratorio de Epidemiología y Salud Pública en Veterinaria, Facultad de Medicina Veterinaria y Zootecnia, Universidad Peruana Cayetano Heredia, Lima, Perú

${ }^{2}$ E-mail: nestorfalcon@upch.pe
}

Recibido: 9 de marzo de 2021

Aceptado para publicación: 18 de septiembre de 2021

Publicado: 27 de octubre de 2021

CLos autores. Este artículo es publicado por la Rev Inv Vet Perú de la Facultad de Medicina Veterinaria, Universidad Nacional Mayor de San Marcos. Este es un artículo de acceso abierto, distribuido bajo los términos de la licencia Creative Commons Atribución 4.0 Internacional (CC BY 4.0) [https:// creativecommons.org/licenses/by/4.0/deed.es] que permite el uso, distribución y reproducción en cualquier medio, siempre que la obra original sea debidamente citada de su fuente original 
personal (79.7\%) y uso de alcohol/desinfectante (60.8\%), en los propietarios. El estudio muestra una baja percepción del riesgo de trasmisión del COVID-19 del ser humano a las mascotas, conocimiento que debe ser reforzado por las autoridades de salud a fin de salvaguardar la relación humano animal basada en prácticas de tenencia responsable de estos animales.

Palabras clave: COVID-19, coronavirus, animales de compañía, una salud, zoonosis

\section{Abstract}

The aim of this study was to evaluate the perception of pet owners about the risk of infection of their pets with COVID-19 in Lima, Peru. A virtual survey was prepared for clients of private veterinary services in Metropolitan Lima. The instrument collected demographic information, knowledge about the transmission of SARS-CoV-2, cases of human infection, handling of the pet during the pandemic and perception of the risk of infection of animals by COVID-19. The survey was answered by 408 pet owners. It was recognized that the main means of transmission of COVID-19 were infected people (93.6\%). Respiratory problems were rarely observed in $\operatorname{dogs}(8.1 \%)$ and cats $(2.2 \%)$. The majority perception of those surveyed was not to relate the respiratory problems of pets with COVID-19 (80.6\%), but they showed concern for the affected health of their animal $(79.7 \%)$. The practice of walking the pets was carried out more than once a day $(38.1 \%)$, being more frequent not using protection for the animals (65.4\%). The walk generally lasted between 10 to 20 minutes (45.4\%), and around the block (46.2\%) and in parks $(36.0 \%)$. Hygiene and prevention measures taken after the walk were bathing and washing of paws $(73.4 \%)$ in pets, and a combination of measures that included social distancing (80.4\%), use of personal protective equipment (79.7\%) and use of alcohol / disinfectant $(60.8 \%)$, in the owners. The study shows a low perception of the risk of transmission of COVID-19 from humans to pets, a knowledge that must be reinforced by health authorities to safeguard the human-animal relationship based on responsible pet ownership practices.

Key words: COVID-19, coronavirus, companion animals, one health, zoonoses

\section{INTRODUCCIÓN}

Los coronavirus pertenecen a la subfamilia Coronavirinae, familia Corona-viridae y orden Nidovirales (Cui et al., 2019) e incluyen cuatro géneros: alfacoronavirus, betacoronavirus, gamacoronavirus y deltacoronavirus (Woo et al., 2012). Su importancia zoonótica se hizo evidente con la presentación del brote del Síndrome Respiratorio Agudo Severo (SARS-CoV) en la provincia de Gunagdong, China, entre los años 20022003 (Drosten et al., 2003; Zhong et al.,
2003) y, posteriormente, con la aparición del Síndrome Respiratorio de Oriente Medio (MERS-CoV) que se reportó en Arabia Saudita y países del medio oriente en 2012 (Zaki et al., 2012).

El 31 de diciembre de 2019 se informó de 27 casos de neumonía de origen desconocido en Wuhan, China y el 7 de enero de 2020 se identificó como agente etiológico a un virus de la familia Coronaviridae. El 30 de enero la Organización Mundial de la Salud(OMS) declaró una emergencia internacional y el 11 de febrero se declaró pandemia a la Enfer- 
medad Infecciosa por Coronavirus (COVID19) y le asignó el nombre de SARS-CoV-2 al virus (OMS, 2020). El primer caso en el Perú se presentó el 6 de marzo de 2020 (MINSA, 2020) y para los primeros días de enero de 2021 se confirmó un acumulado de 1022018 casos con una letalidad de 3.71\% (37925 fallecidos) (MINSA, 2021).

E1 SARS-CoV-2 procedería de una fuente animal de donde se habría diseminado al humano, habiéndose aislado virus relacionados genéticamente en murciélagos Rinolophus (Andersen et al., 2020). Posteriormente, varios países reportaron casos de infección por SARS-CoV-2 en animales (FAO, 2020; FLI, 2020). La Organización Mundial de Sanidad Animal (OIE) informó acerca de la susceptibilidad de animales frente al SARS-CoV-2 indicando que la familia Felidae y Mustelidae presentan una alta susceptibilidad y capacidad de infectar individuos de su misma especie (OIE, 2020a).

Dada la transmisión antropozoonótica en gatos (Zhang et al., 2020), perros (Sit et al., 2020), tigres, leones (USDA, 2020a) y visones (Oreshkova et al., 2020), se sospecha que los animales podrían estar involucrados en la pandemia de COVID-19, aunque no se ha demostrado la transmisión natural de animales a humanos (Abdel-Moneim y Abdelwhab, 2020). Sin embargo, la presentación de casos de COVID-19 en animales habría causado preocupación e incertidumbre que representa una amenaza para el bienestar de las mascotas. El asociar a ellos con la propagación del virus SARS-CoV-2 podría hacer que propietarios temerosos abandonen o sacrifiquen a perros y gatos por el temor a infectarse. En este contexto, el objetivo del estudio fue evaluar la percepción de los propietarios de mascotas acerca del riesgo de infección de sus mascotas con COVID-19 en Lima, Perú.

\section{Materiales y Métodoos}

El estudio observacional y descriptivo se desarrolló en la ciudad de Lima Metropolitana entre noviembre y diciembre de 2020 . La población objetivo estuvo compuesta por propietarios de mascotas de varios distritos de la capital, a los que se tuvo acceso solicitando apoyo a médicos veterinarios que laboraban en clínicas o consultorios para que hagan llegar las encuestas vía electrónica a su cartera de clientes. Las personas que participaron en la encuesta fueron de ambos sexos y adultas.

El tamaño de muestra fue definido mediante la fórmula de comprobación de una proporción para poblaciones desconocidas. Se utilizó un nivel de confianza del $95 \%$, error máximo admisible del $5 \%$ y una proporción referencial de $50 \%$ para la variable percepción de los propietarios de que el COVID-19 se puede transmitir del hombre a los animales (valor utilizado cuando se desconoce una proporción referencial). El tamaño de muestra calculado fue de 385 encuestas.

El instrumento de recolección de información incluyó las siguientes secciones:

- Información demográfica del encuestado: sexo (masculino, femenino), grupo etario (18-29, 30-59, |>59 años), grado de instrucción (escolar, superior), habitantes de la vivienda $(1-2,3-5,>5$ personas), procedencia según distribución del Instituto Nacional de Estadística e Informática (INEI, 2014) (Lima Norte, Lima Centro, Lima Este Lima Sur)) y tenencia de mascota (perro, gato, ambos),

- Conocimiento sobre reservorios animales y forma de transmisión del virus SARS-COV-2.

- Información sobre casos de COVID-19 humana en familiares del propietario de mascotas que compartían la vivienda, 
- Información del manejo de las mascotas intra y extradomiciliario (incluye medidas de bioseguridad durante los paseos), - Percepción acerca del riesgo de exposición al COVID-19 según actividad realizada con la mascota o la convivencia con casos humanos de COVID-19.

La encuesta fue evaluada mediante juicio de expertos por dos médicos veterinarios y una licenciada en psicología comunicacional. El instrumento fue modificado de acuerdo con las observaciones de los evaluadores y se consideró como versión final aquella que fue aprobada por unanimidad. Después de ello, la encuesta fue llevada a un formato virtual utilizando el recurso de la plataforma Google (formularios virtuales Google).

La encuesta en formato virtual incluyó un encabezado de invitación al propietario de la mascota en el que se incluía además el objetivo principal del estudio, un resumen del consentimiento informado, la declaración jurada de mayoría de edad y las indicaciones para el llenado de la encuesta. El enlace de la encuesta virtual fue enviado a médicos veterinarios que se comprometieron a apoyar el estudio difundiendo la misma entre sus clientes a través de medios electrónicos (WhatsApp, messenger o correo electrónico). Se recomendó evitar el reenvío de la encuesta a terceras personas, con la finalidad de tener una mayor confianza de que los resultados que se obtengan provengan de la población objetivo.

La información obtenida se almacenó en una hoja de Microsoft Excel. Los resultados se analizaron mediante estadística descriptiva. Para la variable cuantitativa edad se utilizó medidas de tendencia central y dispersión. En caso de las variables cualitativas nominales (sexo, grado de instrucción, procedencia, tenencia de animales, medios de transmisión, síntomas, diagnóstico, experiencias, percepciones y medidas preventivas) y ordinales (grupo etario, habitantes en la vi- vienda, frecuencia y tiempo de paseo) se utilizaron valores de frecuencia absoluta y relativa. La asociación entre las variables demográficas y las percepciones con relación al riesgo de infección de las mascotas con COVID-19 se analizó mediante la prueba de Chi Cuadrado. El análisis estadístico se realizó utilizando el programa estadístico Stata 15.0.

La participación en el estudio fue voluntaria y las encuestas fueron anónimas. Los encuestados se encontraron en libertad de negarse a responder aquellas preguntas que estimaban conveniente o de retirarse del estudio, para lo cual solo debían de evitar el envío de las respuestas a través del enlace digital alcanzado. El estudio fue aprobado por el Comité Institucional de Ética de la Universidad Peruana Cayetano Heredia con la constancia N. ${ }^{\circ}$ 631-36-20.

\section{Resultados}

El estudio obtuvo 408 encuestas válidas. La edad de los encuestados fue de $34.0 \pm$ 11.6 años (rango de 18 a 77). Predominaron encuestados de sexo femenino, grado de instrucción superior, que compartían vivienda entre 3 a 5 personas, procedían de los distritos de Lima Centro y la mayoría poseía un perro como mascota (Cuadro 1).

El medio de transmisión del COVID-19 al humano, reconocido con mayor frecuencia fue a partir de las personas (Cuadro 2). Solo el 31.4\% (128) de encuestados mencionó que el virus COVID-19 podía ser transmitido de una persona infectada a la mascota. El 29.9\% (122) afirmó que al menos una persona con quien compartía la vivienda habría sido infectada por el COVID-19. En este grupo, los signos clínicos observados con mayor frecuencia fueron compatibles con gripe o resfriado y el diagnóstico fue realizado con mayor frecuencia mediante pruebas rápidas (Cuadro 3). 
Cuadro 1. Características demográficas de los encuestados sobre riesgo de infección con COVID-19 en Lima - Perú $(n=408)$

\begin{tabular}{lcc}
\hline \multicolumn{1}{c}{ Variable } & $\mathrm{n}$ & $\%$ \\
\hline Sexo & & \\
$\quad$ Femenino & 291 & 71.3 \\
$\quad$ Masculino & 117 & 28.7 \\
Grupo etario (años) & & \\
$\quad 18$ a 29 & 184 & 45.1 \\
30 a 59 & 212 & 52.0 \\
$\quad>59$ & 12 & 2.9 \\
Grado de instrucción & & \\
$\quad$ Escolar & 35 & 8.6 \\
Superior & 373 & 91.4 \\
Habitantes en la vivienda & & \\
1 a 2 & 62 & 15.2 \\
3 a 5 & 252 & 61.8 \\
$\quad>5$ & 94 & 23.0 \\
Procedencia & & \\
Lima Centro & 150 & 36.8 \\
Lima Norte & 103 & 25.2 \\
Lima Este & 102 & 25.0 \\
Lima Sur & 53 & 13.0 \\
Tenencia de mascotas & & \\
Perros & 254 & 62.3 \\
Gatos & 62 & 15.2 \\
Ambos & 92 & 22.5 \\
\hline
\end{tabular}

E1 $10.8 \%$ (44) mencionó que sus mascotas presentaron signos clínicos respiratorios durante el periodo de la pandemia por COVID-19. De ellos, 75\% (33) fue a visitar al Médico Veterinario, 20.5\% (9) no recibió tratamiento y $4.5 \%$ (2) ofrecieron tratamiento casero. De los propietarios cuyos animales presentaron signos clínicos respiratorios, $14.5 \%$ (18) mencionó haber tenido un caso de COVID-19 en la familia. En general, $79.2 \%$ (323) de los entrevistados mencionaron que durante la pandemia siguieron visitando al médico veterinario para la atención de sus animales. La percepción de los encuestados en general acerca de su reacción ante la eventual presentación de una afección respiratoria en sus mascotas durante el periodo de pandemia COVID-19 fue de preocupación, aunque su aparición no la relacionaban con COVID-19 (Cuadro 4).

El 70.1\% (286) de los encuestados mencionó que paseaba a su mascota durante el periodo de pandemia por COVID-19, siendo lo más frecuente el paseo diario y más de una vez al día por 10 a 20 minutos. La mayoría no consideró ningún medio de protección del animal para el paseo, siendo llevados a pasear alrededor de la manzana y a los parques (Cuadro 5).

La medida preventiva de mayor frecuencia en caso la mascota hubiera estado en contacto con una persona sospechosa de estar infectada con COVID-19 fue el baño y lavado de patas. En caso de las personas, las medidas principales para evitar estos contactos fueron el distanciamiento social, uso de equipos de protección personal (mascarillas y protector facial) y el uso de alcohol o desinfectantes (Cuadro 6). La percepción de los encuestados acerca de evitar el contacto de las mascotas con pacientes infectados y/o enfermos con COVID-19 fue afirmativa para el $62.3 \%$ (254) en tanto que $37.7 \%$ (154) no lo consideró necesario.

\section{Discusión}

La mayor proporción de encuestados fueron mujeres, lo que puede estar relacionado a su mayor interés por las mascotas. El estudio de Shaw (2012) sobre la comunicación entre veterinarios y clientes, observó un sesgo femenino que demuestra que las mujeres se encuentran más comprometidas con los problemas relacionados a su mascota. El grupo etario estuvo comprendido principalmente por personas entre 30 a 59 años, seguido de jóvenes de 18 a 29 años y de grado 
Cuadro 2. Conocimiento de los encuestados sobre la forma de transmisión del virus del COVID-19. Lima, Perú, 2020 ( $\mathrm{n}=408)$

\begin{tabular}{lcc}
\hline Medio de transmisión & $\mathrm{n}$ & $\%$ \\
\hline Personas infectadas & 382 & 93.6 \\
Superficies contaminadas & 269 & 65.9 \\
Alimentos contaminados & 152 & 37.3 \\
Animales infectados & 36 & 8.8 \\
\hline
\end{tabular}

Los encuestados podían ofrecer más de una respuesta

Cuadro 3. Síntomas y diagnóstico de COVID-19 en personas infectadas con quien compartía la vivienda el encuestado. Lima, Perú $(\mathrm{n}=122)$

\begin{tabular}{lcc}
\hline Variable & $\mathrm{n}$ & $\%$ \\
\hline Síntomas de COVID-19 & 3 & \\
Asintomático & 21 & 2.5 \\
Dolor de cabeza pasajero & 57 & 17.2 \\
Signos compatibles con gripe & 23 & 46.7 \\
Dificultad respiratoria leve a moderada & 10 & 18.9 \\
Dificultad respiratoria con hospitalización & 8 & 8.2 \\
Dificultad respiratoria con ingreso a UCI & & 6.6 \\
Diagnóstico de COVID-19 & 66 & \\
Prueba rápida & 28 & 54.1 \\
Prueba rápida y molecular & 16 & 23.0 \\
Prueba molecular & 12 & 13.1 \\
Sintomatología y examen (Rx o tomografia) & 9.8 \\
\hline
\end{tabular}

de instrucción superior. Ello se relacionaría a que profesionales y personas pertenecientes a la población económicamente activa serían quienes usualmente llevan a sus mascotas a los servicios veterinarios, servicios que fueron considerados como puntos de muestreo en el estudio.

Un alto porcentaje de encuestados consideró que la principal vía de transmisión del virus SARS-CoV-2 era el contacto con personas infectadas. Ello estaría relacionado a la información difundida por los medios de comunicación a los que tendrían acceso los encuestados. Resultados similares encuentran Saqlain et al. (2020) en su estudio con personas primariamente de grado de instrucción superior, encontrando que más del $90 \%$ presentaban un conocimiento satisfactorio sobre el tema.

Aun cuando los conocimientos respecto del comportamiento del COVID-19 se han ido estandarizando, aún existe un pequeño 
Cuadro 4. Percepciones con relación a problemas respiratorios en mascotas durante el periodo de pandemia COVID-19. Lima, Perú $(\mathrm{n}=408)$

\begin{tabular}{lcc}
\hline \multicolumn{1}{c}{ Variable } & $\mathrm{n}$ & $\%$ \\
\hline Respuesta emocional $^{1}$ & & \\
Preocupación & 325 & 79.7 \\
Tristeza & 142 & 34.8 \\
Miedo & 102 & 25.0 \\
Ninguna emoción & 36 & 8.8 \\
Relacionar con COVID-19 & \\
No & 329 & 80.6 \\
Sí & 79 & 19.4 \\
\hline${ }^{1}$ Los encuestados podían ofrecer más de una \\
respuesta
\end{tabular}

porcentaje de encuestados que considera que esta enfermedad se puede transmitir por contacto directo con animales enfermos, situación que se debería a que a inicios de la pandemia se difundieron noticias referentes a infecciones de SARS-CoV-2 en perros y gatos (OIE, 2020b; Goumenou et al., 2020). Ante ello, el Centro para el Control y Prevención de Enfermedades (CDC, 2020) emitió comunicados aclarando que el riesgo de infección animal-humano es bajo y exhorta considerar a las mascotas como un miembro de la familia, con la finalidad de tomar las medidas de tenencia responsable adecuadas.

La presencia de casos humanos de COVID-19 podría ser considerada como fuente de exposición para los animales. Los signos clínicos que presentaron los afectados en el núcleo familiar eran predominantemente compatibles con gripe, seguido de una dificultad respiratoria de leve a moderada; por lo que no habrían sido considerados importantes en el entorno de la población en estudio, que además en su mayoría no pertenecía a grupos etarios de riesgo (Mendoza-Ticona et al., 2020). El método diagnóstico utilizado con mayor frecuencia fue la Prueba Rápida, debido a que en la primera etapa de la pandemia era la herramienta que disponía el Ministerio de Salud para la detección de exposición (anticuerpos) (MINSA, 2021).

Se presentaron pocos casos de cuadros respiratorios en los perros y gatos de la población en estudio, resultando favorable que la mayoría de encuestados no lo considere relacionado a casos de COVID-19. Estos cuadros respiratorios pudieron estar asociados a infecciones por otros agentes patógenos como: Herpesvirus Felino, Calicivirus Felino (Dawson et al., 2004) y Coronavirus Canino (MacLachlan y Dubovi, 2011), siendo este último, causante de cuadros entéricos (Berrezueta, 2014) que también se observan en infecciones por COVID-19 en personas (Schmulson et al., 2020). Por otra parte, estudios experimentales han demostrado que los perros presentan susceptibilidad baja frente a infección por SARS-CoV-2 (Shi et al., 2020), y los gatos presentan sintomatología variable desde casos asintomáticos a moderados, con transmisión a otros felinos domésticos (Halfmann et al., 2020; Shi et al., 2020). Por otro lado, se debe tener presente que la búsqueda de COVID-19 en mascotas no es una práctica frecuente y se ha limitado mayormente a países como Bélgica y Estados Unidos donde disponen de pruebas moleculares específicas para mascotas (OIE, 2020b; Thompson, 2020; USDA, 2020b).

Ante la observación de una afección respiratoria, los propietarios optaron principalmente por la visita a un médico veterinario. Ello es una práctica importante, ya que, dentro del marco de la pandemia, la OPS (2020) exhorta a llamar y acudir al Médico Veterinario u otro funcionario de salud pública para levantar el informe correspondiente respecto a enfermedades atípicas o si el animal entró en contacto con una persona sospechosa de COVID-19. Sin embargo, se debe de tener en cuenta que en el Perú no se han desarrollado protocolos para este fin y que el recurrir a la atención veterinaria está relacionado principalmente a la preocupación por la salud de las mascotas. 
Cuadro 5. Prácticas de paseo de la mascota durante el periodo de la pandemia por COVID19. Lima, Perú $(\mathrm{n}=286)$

\begin{tabular}{lcc}
\hline \multicolumn{1}{c}{ Variable } & $\mathrm{n}$ & $\%$ \\
\hline Frecuencia paseo de mascota & & \\
Paseo diario $>$ 1 vez & 109 & 38.1 \\
Paseo diario 1 vez & 77 & 26.9 \\
1-2 veces/semana & 76 & 26.6 \\
3-4 veces/semana & 24 & 8.4 \\
Medio de protección durante paseo & & \\
No usa ningún medio de protección & 187 & 65.4 \\
Accesorios (collar, correa, collar isabelino & 61 & 21.3 \\
Accesorios de limpieza & 19 & 6.6 \\
Lavado de patas (agua) & 19 & 6.6 \\
Tiempo de paseo (minutos) & & \\
$<10$ & 68 & 23.8 \\
10-20 & 130 & 45.4 \\
>20 & 88 & 30.8 \\
Lugar usual para paseo & & \\
Alrededor de la manzana & 132 & 46.2 \\
Parques & 103 & 36.0 \\
Puerta de la casa & 47 & 16.4 \\
Mercado & 4 & 1.4 \\
\hline
\end{tabular}

Cuadro 6. Prácticas de prevención relacionadas al contacto con personas sospechosas con COVID-19. Lima, Perú $(\mathrm{n}=286)$

\begin{tabular}{lcc}
\hline \multicolumn{1}{c}{ Variable } & $\mathrm{n}$ & $\%$ \\
\hline Prevención de las mascotas pos-paseo & & \\
Baños y lavado de patas & 210 & 73.4 \\
No tomaba medidas & 38 & 13.3 \\
Desinfección & 35 & 12.2 \\
Consulta a médico veterinario & 3 & 0.9 \\
Prevención del propietario durante paseo ${ }^{(*)}$ & & \\
Distanciamiento social & 230 & 80.4 \\
Equipos de protección & 228 & 79.7 \\
Uso alcohol/desinfectante & 174 & 60.8 \\
No previene - evita pasear a la mascota & 3 & 0.9 \\
\hline
\end{tabular}

${ }^{1}$ Los encuestados podían ofrecer más de una respuesta 
En general, la mayoría de los encuestados mencionaron que ante la eventualidad de que las mascotas presenten signos clínicos respiratorios, estos signos no estarían relacionados con el COVID-19. En contraste, existen notificaciones de otros países donde algunos propietarios relacionaron la presencia de estos signos clínicos con la infección por SARS-CoV-2 y optaron por el abandono o sacrificio de la mascota (OPS, 2020).

A pesar de no existir evidencia científica suficiente que compruebe la infección y transmisión del SARS-CoV-2 en perros y gatos, se recomienda mantener las medidas de higiene y bioseguridad, tomando en cuenta que el SARS-CoV-2 ha presentado varias cepas a nivel mundial y comparte ciertos epítopos con algunos virus relacionados taxonómicamente y con tropismo por los animales sinantrópicos (Costagliola et al., 2021). El estudio de Zhao et al. (2021) evaluó 910 perros de Wuhan mediante un ELISA indirecto, obteniendo una prevalencia de $1.8 \%$.

La principal frecuencia de paseo de los perros fue diaria, probablemente asociado a la costumbre de desarrollar esta práctica en forma rutinaria antes de presentarse las restricciones de movilidad producto de la pandemia. Se ha recomendado a las personas que durante los paseos no se intente socializar y que se debe de priorizar los horarios con poca afluencia de personas (da Mansilla, 2020), ello mismo es extensivo a los paseos de mascotas. Además, atendiendo los criterios establecidos por el Colegio Médico Veterinario del Perú, se sugiere que las mascotas sean paseadas por una persona que no pertenezca a una población de riesgo (Aguilar y Falcón, 2020). Por otro lado, considerando las consecuencias que produce el aislamiento en este periodo de pandemia, el paseo de las mascotas es importante para preservar la buena salud mental de los propietarios, además de ayudar a contrarrestar la depresión y ansiedad ocasionadas por la pandemia y el aislamiento (Morgan et al., 2020).
Una proporción mayoritaria de propietarios encuestados comentó que durante el paseo no usaban ningún medio de protección para su mascota, resultado que se relaciona con la percepción de los encuestados que descartan la posibilidad de una infección por COVID-19 en los animales. Este hecho es favorable y se sustenta en las comunicaciones de organismos técnicos como la OIE (2020a). Sin embargo es importante que los animales salgan bajo control, por lo que el uso de accesorios de sujeción como collares y arneses impide el acercamiento del animal a terceras personas (Aguilar y Falcón, 2020).

El tiempo de paseo de las mascotas osciló mayormente entre 10 a 20 minutos, periodo que habría sido menor al que invirtieran antes de la pandemia. Al respecto, Bowen et al. (2020) hallaron que el $49.7 \%$ de propietarios en poblaciones de España redujo el tiempo de paseo de las mascotas a menos de 30 minutos durante el periodo de confinamiento debido a las sugerencias brindadas por las autoridades sanitarias. Respecto a la zona de caminata, los encuestados optaron por los alrededores de la manzana y los parques, lugares que probablemente fueron elegidos por ser espacios abiertos ya conocidos por los animales y en donde se habría solido llevar para satisfacer las necesidades fisiológicas de la mascota.

Los baños y lavado de las patas de los animales fueron las prácticas realizadas con más frecuencia por los propietarios como medidas preventivas después del paseo. Estas también son indicadas por la OPS (2020), recomendando el uso de agua y jabón neutro, asegurando la limpieza del pelo y entre los dedos. Sin embargo, una proporción menor de encuestados mencionó usar alcohol y desinfectantes en las patas, medidas no recomendables debido a que pueden causar irritación en la piel. Es recomendable que ante la duda, los propietarios busquen asesoría de un Médico Veterinario con la finalidad de conocer los productos para prevenir la participación de las mascotas en la movilización del virus SARS-CoV-2 (OPS, 2020). 
La pandemia de COVID-19 ha generado incertidumbre, preocupación y ha afectado fuertemente a la población humana. A ello se ha sumado algunas dudas acerca del rol de los perros y gatos en la epidemiología de COVID-19. Frente a esta situación, esta investigación brinda información sobre la percepción de los propietarios de mascotas ajustada a la realidad peruana, que debe de fortalecer los reportes de las autoridades sanitarias sobre el rol de la mascota en la transmisión del SARS-CoV-2 al ser humano. Este riesgo es considerado bajo por los propietarios de los animales, permitiendo de esta manera salvaguardar la salud humana y el bienestar animal, sin descuidar las prácticas de tenencia responsable de las mascotas.

\section{Conclusiones}

- El medio de transmisión de COVID-19 reconocido con mayor frecuencia por los encuestados fue el contacto con personas infectadas.

- La observación de signos clínicos respiratorios en las mascotas no fue relacionada con la presencia de COVID-19 y las visitas al médico veterinario era por preocupación por la salud del animal.

- Los propietarios paseaban a sus mascotas con una frecuencia diaria y más de una vez, no utilizando ningún tipo de protección para los animales, pero sí elementos de sujeción. El tiempo de paseo de la mascota más frecuente fue de 10 a 20 minutos y se realizaba principalmente alrededor de la manzana y en parques.

- La medida de prevención de infección o contaminación por COVID-19 en las mascotas adoptada con mayor frecuencia por los propietarios fue el baño y lavado de patas, y en el caso de las personas fueron el distanciamiento social, uso de equipos de protección personal y el uso de alcohol/desinfectante.
Literatura Citada

1. Abdel-Moneim AS, Abdelwhab EM. 2020. Evidence for SARS-CoV-2 infection of animal hosts. Pathogens 9: 529. doi: 10.3390/pathogens 9070529 .

2. Aguilar S, Falcón N. 2020. E1 COVID-19 y los animales de compañía: Una breve revisión. MV Rev Cien Vet 36: 5-13.

3. Andersen K, Rambaut A, Lipkin W, Holmes E, Garry R. 2020. The proximal origin of SARS-CoV-2. Nat Med 26: 450-452.

4. Berrezueta JE. 2014. Diagnóstico de coronavirus canino mediante la prueba de ELISA, en el Cantón El Guabo. Tesis de Médico Veterinario Zootecnista. Machala: Univ. Técnica de Machala. 51 p.

5. Bowen J, García E, Darder P, Argüelles J, Fatjó J. 2020. The effects of the Spanish COVID-19 lockdown on people, their pets and the human-animal bond. J Vet Behav 40:75-91. doi: 10.1016/j.jveb.2020.05.013

6. [CDC] Centro para el Control y Prevención de Enfermedades. 2020. What you should know about COVID19 and pets. [Internet]. Disponible en: https://espanol.cdc.gov/coronavirus/ 2019-ncov/daily-life-coping/pets.html

7. Costagliola A, Liguori G, d'Angelo D, Costa C, Ciani F, Giordano A. 2021. Do animals play a role in the transmission of severe acute respiratory syndrome coronavirus-2 (SARS-CoV2)? A commentary. Animals 11: 16. doi: 10.3390/ani11010016

8. Cui J, Li F, Shi Z. 2019. Origin and evolution of pathogenic coronaviruses. Nat Rev Microbiol 17: 181-192. doi: 10.1038/s41579-018-0118-9

9. Da Mansilla G 2020. Clínicas veterinarias y paseos con animales de compañía ante el COVID-19: una actividad esencial para el bienestar del animal como ser sintiente. Derecho Animal 11: 17-29. doi: $10.5565 / \mathrm{rev} / \mathrm{da} .499$ 
10. Dawson S, Radford A, Gaskel L. 2004. Clinical update on feline respiratory pathogens. In Pract 26: 320323.

11. Drosten C, Gunther S, Preiser W, van der Werf $S$, Brodt HR, Becker S, Rabenau H, et al. 2003. Identification of a novel coronavirus in patients with severe acute respiratory syndrome. New Engl J Med 348: 1967-1976. doi: 10.1056/ NEJMoa030747

12. [FAO] Organización de las Naciones Unidas para la Alimentación y la Agricultura. 2020. Exposure of humans or animals to sars-cov-2 from wild, livestock, companion and aquatic animals. Rome: FAO. Informe Técnico. 38 p.

13. [FLI] Friedrich Loeffler Institute. 2020. Novel Coronavirus SARS-CoV2: Fruit bats and ferrets are susceptible; pigs and chickens are not. [Internet]. Available in:https://www.fli.de/en/press/ press-releases/press-singleview/novelcoronavirus-sars-cov-2-fruit-bats-andferrets-are-susceptible-pigs-andchickens-are-not/

14. Goumenou M, Spandidos D, Tsatsakis A. 2020. Possibility of transmission through dogs being a contributing factor to the extreme Covid 19 outbreak in North Italy. Mol Med Rep 21: 2293-2295. doi: 10.3892/ mmr.2020.11037

15. Halfmann P, Hatta M, Chiba S, Maemura T, Fan S, Takeda M, Kinoshita N, et al. 2020. Transmission of SARS-CoV-2 in domestic cats. New Engl J Med 383: 592-594. doi: 10.1056/ NEJMc2013400

16. [INEI] Instituto Nacional de Estadística e Informática. 2014. Una mirada a Lima Metropolitana. 81 p. [Internet] Disponible en: https://www.inei.gob.pe/ media/MenuRecursivo/publicaciones_digitales/Est/Lib1168/libro.pdf

17. MacLachlan N, Dubovi E. 2011. Fenner's veterinary virology. $4^{\circ}$ ed. Elsevier. $491 \mathrm{p}$.
18. Mendoza-Ticona A, Valencia G, Quintana A, Cerpa B, García G, Álvarez $C$, Rivero J. 2020. Clasificación clínica y tratamiento temprano de la COVID19. Reporte de casos del Hospital de Emergencias Villa El Salvador, LimaPerú. Acta Med Peru 37: 186-191.

19. [MINSA] Ministerio de Salud. 2020. COVID-19 Comunicado $\mathrm{N}^{\circ} 147$. [Internet]. Disponible en: https:// www.gob.pe/institucion/minsa/noticias/ 189091-minsa casos-confirmados-porcoronavirus-COVID-19-ascienden-a272-364-en-el-peru-comunicado-n 147.

20. [MINSA] Ministerio de Salud. 2021. Casos confirmados por coronavirus Covid-19 ascienden a 1022018 en el Perú (Comunicado N. ${ }^{\circ} 379$ ). [Internet]. Disponible en: https://www.gob.pe/ institucion/minsa/noticias/323555-minsacasos-confirmados-por-coronaviruscovid-19-ascienden-a-1-022-018-en-elperu-comunicado-n-379/

21. Morgan L, Protopopova A, Birkler R, Itin-Shwartz, Sutton G, Gamliel A, Yakobson B, et al. 2020. Human-dog relationships during the COVID-19 pandemic: booming dog adoption during social isolation. Human Soc Sci Com 7: 155.

22. [OIE] World Organisation for Animal Health. 2020a. Infection with SARSCOV-2 in animals. Paris: OIE. [Internet]. Available in: https://www.oie.int/filead$\mathrm{min} /$ Home/MM/EN_Factsheet_SARSCoV-2.pdf

23. [OIE] World Organisation for Animal Health. 2020b. Information provided by the National Veterinary Services of Belgium. [Internet]. Available in: https:// www.oie.int/fileadmin/Home/eng/ Our_scientific expertise/docs/pdf/COV19/Belgium_28.03.20.pdf

24. [OMS] Organización Mundial de la Salud. 2020. COVID-19: Cronología de la actuación de la OMS. [Internet]. Disponible en: https://www.who.int/es/ news-room/detail/27-04-2020-whotimeline-COVID-19. 
25. [OPS] Organización Panamericana de la Salud. 2020. La COVID19 Comunicado conjunto sobre el nuevo Coronavirus y los perros y gatos. Washington: OPS. [Internet]. Disponible en: https://www.paho.org/es/documentos/ covid-19-comunicado-conjunto-sobrenuevo-coronavirus-perros-gatos.

26. Oreshkova N, Molenaar R, Vreman $S$, Harders F, Oude B, Hakze-van der Honing R, Gerhards N, et al. 2020. SARS-CoV-2 infection in farmed minks, The Netherlands, April and May 2020. Eurosurveillance 25: 2001005. doi: 10.2807/1560-7917.ES.2020.25.23.2001005

27. Saqlain M, Munir M, Rehman S, Gulzar A, Naz S, Ahmed Z, Tahir A, et al. 2020. Knowledge, attitude, practice and perceived barriers among healthcare workers regarding COVID19: a crosssectional survey from Pakistan. J Hosp Infect 105: 419-423. doi: 10.1016/j.jhin.2020.05.007

28. Schmulson M, Dávalos M, Berumen J. 2020. Alerta: los síntomas gastrointestinales podrían ser una manifestación de la COVID-19. Rev Gastroenterol Mex 85: 282-287. doi: 10.1016/ j.rgmx.2020.04.001

29. Shaw J, Bonnett B, Roter D, Adams $C$, Larson S. 2012. Gender differences in veterinarian client-patient communication in companion animal practice. $\mathrm{J}$ Am Vet Med Assoc 241: 81-88. doi: 10.2460/javma.241.1.81

30. Shi J, Wen Z, Zhong G, Yang H, Wang C, Huang B, Liu R, et al. 2020. Susceptibility of ferrets, cats, dogs, and other domesticated animals to SARScoronavirus 2. Science 368: 1016-1020. doi: $10.1126 /$ science.abb7015

31. Sit T, Brackman C, Ip S, Tam K, Law P, To E,Yu V, et al. 2020. Infection of dog with SARS-CoV-2. Nature 586: 776-778.

32. Thompson H. 2020. A cat appears to have caught the coronavirus, but it's complicated. [Internet]. Disponible en: https://www.sciencenews.org/article/ cats-animals-pets-coronavirus-covid19.
33. [USDA] Animal and Plant Health Inspection Service. 2020a. Statement on the confirmation of COVID-19 in a tiger in New York. [Internet]. https:// www.aphis.usda.gov/aphis/newsroom/ news/sa_by_date/sa-2020/NY-zooCOVID-19

34. [USDA]. United States Department of Agriculture. 2020b. SARS-CoV-2 in animals in The United States. [Internet]. Disponible en: https://www.aphis.usda.gov/aphis/ourfocus/animalhealth/ SA_One_Health/sars-cov-2-animals-us

35. Woo PC, Lau SK, Lam CS, Lau CC, Tsang AK, Lau JH, Bai R, et al. 20212. Discovery of seven novel mammalian and avian coronaviruses in the genus deltacoronavirus supports bat coronaviruses as the gene source of alphacoronavirus and betacoronavirus and avian coronaviruses as the gene source of gammacoronavirus and deltacoronavirus. J Virol 86: 3995-4008. doi: 10.1128/JVI.06540-11

36. Zaki A, van Boheemen S, Bestebroer T, Osterhaus A, Fouchier R. 2012. Isolation of a novel coronavirus from a man with pneumonia in Saudi Arabia. New Engl J Med 367: 1814-1820. doi: 10.1056/NEJMoa1211721

37. Zhang Q, Zhang H, Huang K, Yang Y, Hui X, Gao J, He X, et al. 2020. SARS-CoV-2 neutralizing serum antibodies in cats: a serological investigation. BioRxiv. [Internet]. Disponible en: https://www.biorxiv.org/content/ 10.1101/2020.04.01.021196v1.

38. Zhao Y, Yang Y, Gao J, Huang $\mathrm{K}$, Hu C, Hui X, He X, et al. 2021. A serological survey of severe acute respiratory syndrome coronavirus 2 in dogs in Wuhan. Transbound Emerg Dis 7: 10.1111/tbed.14024. doi: 10.1111/ tbed.14024

39. Zhong NS, Zheng BJ, Li YM, Poon, Xie ZH, Chan KH, Li PH, et al. 2003. Epidemiology and cause of severe acute respiratory syndrome (SARS) in Guangdong, People's Republic of China, in February, 2003. Lancet 362: 1353-1358. doi: 10.1016/s0140-6736(03)14630-2. 